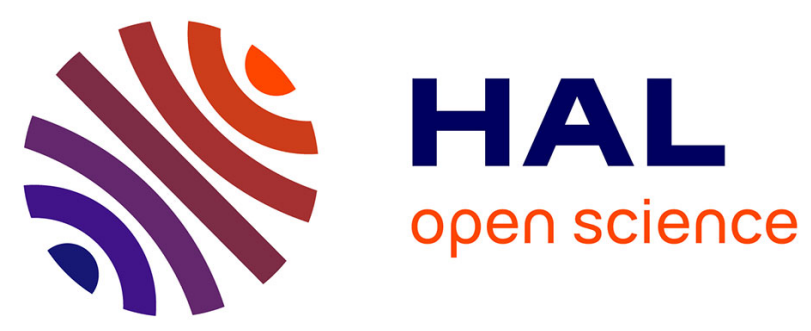

\title{
Place de la préservation de la fertilité dans le parcours de transition des femmes transgenres
}

\author{
Emmanuelle Benaloun, Nathalie Sermondade, Emilie Moreau, Nathalie \\ Chabbert-Buffet, Sarra Cristofari, Nicolaï Johnson, Rachel Lévy, Charlotte \\ Dupont
}

\section{To cite this version:}

Emmanuelle Benaloun, Nathalie Sermondade, Emilie Moreau, Nathalie Chabbert-Buffet, Sarra Cristofari, et al.. Place de la préservation de la fertilité dans le parcours de transition des femmes transgenres. Gynécologie Obstétrique Fertilité \& Sénologie, 2021, 49 (6), pp.547-552. 10.1016/j.gofs.2021.01.007. hal-03263387

\section{HAL Id: hal-03263387 \\ https://hal.sorbonne-universite.fr/hal-03263387}

Submitted on 17 Jun 2021

HAL is a multi-disciplinary open access archive for the deposit and dissemination of scientific research documents, whether they are published or not. The documents may come from teaching and research institutions in France or abroad, or from public or private research centers.
L'archive ouverte pluridisciplinaire HAL, est destinée au dépôt et à la diffusion de documents scientifiques de niveau recherche, publiés ou non, émanant des établissements d'enseignement et de recherche français ou étrangers, des laboratoires publics ou privés. 
7 Emmanuelle Benaloun ${ }^{1}$, Nathalie Sermondade ${ }^{2}$, Emilie Moreau $^{1}$, Nathalie Chabbert-Buffet ${ }^{3}$,

$8 \quad$ Sarra Cristofari ${ }^{4}$, Nicolaï Johnson ${ }^{3}$, Rachel Lévy ${ }^{2}$, Charlotte Dupont ${ }^{2}$

9

10 19 AP-HP, Hôpital Tenon, service de Biologie de la Reproduction CECOS, Paris, France.

$11{ }^{2}$ Sorbonne Université, Saint Antoine Research center, INSERM équipe Lipodystrophies génétiques et acquises.

12 Service de biologie de la reproduction-CECOS, AP-HP, Hôpital Tenon, Paris, France

$13{ }^{3}$ AP-HP, Hôpital Tenon, Service de Gynécologie-Obstétrique et Médecine de La Reproduction, Paris, France. 14

$15{ }^{4}$ AP-HP, Hôpital Tenon, Service de chirurugie plastique reconstructrice et esthetique, Sorbonne université, 16 Paris, France.

17

19 Les auteurs ne déclarent aucun conflit d'intérêt lié à cet article 


\section{Introduction}

Définitions : Transidentité et dysphorie de genre

Le genre d'un individu est généralement assigné à la naissance en fonction de l'anatomie, un caryotype s'avérant nécessaire dans certaines situations. Pour la plupart des individus, cette attribution de genre correspond à l'identité de genre, c'est-à-dire à l'identification de soi en tant qu'homme ou femme. Néanmoins, certains enfants/adolescents/adultes peuvent être confrontés à une incongruence du genre assigné et s'identifier dans un autre genre. La dysphorie de genre est le terme médical utilisé par le DSM-5 (manuel diagnostique et statistique des troubles mentaux V5) pour décrire la souffrance ou la détresse causée par "l'incongruité marquée entre leur genre vécu ou exprimé et celui qui leur a été attribué à la naissance". Les termes liés à la transidentité sont décrits dans le tableau 1. Aux États-Unis en 2016, il a été estimé que 0,6 à 0,7\% des adultes se définissaient comme transgenres [1]. En Europe, des professionnels estiment une prévalence de $1 / 20000$ qui pourrait être dix fois plus importante dans la population générale [2]. La conscience de ne pas être dans le «bon » genre peut être vécue très tôt par les enfants, certains enfants ressentant une incongruence de genre dès l'âge de 8,3 +/- 4,5 ans [3]. Longtemps utilisé, le terme «transsexuel », dérivé de transsexualisme, a été développé par le monde médical au XIXème siècle pour désigner la «maladie mentale » dont souffraient les personnes transgenres. Depuis, il a été démontré que la transidentité n'est pas une pathologie et l'Organisation Mondiale de la Santé (OMS) l'a donc retirée des maladies mentales en 2019. Le terme transexuel ne doit plus être utilisé. 
L'accès aux soins et la reconnaissance de l'identité de genre est très variable selon les pays.

47 Certains continuent d'appliquer une très forte discrimination envers les personnes transgenres.

La plupart pays reconnaissent les deux identités de genre traditionnelles, mais ont tendance à exclure les autres identités et expressions de genre. Certains pays reconnaissent néanmoins un « troisième sexe ».

En France, il est possible pour une personne transgenre de modifier son prénom et son état civil.

La loi de modernisation de la Justice du XXIème siècle du 18 novembre 2016 a réformé les conditions du changement d'état civil. Ces modifications se font en deux étapes. Généralement, le prénom est modifié en premier. La démarche se fait en mairie auprès de l'officier d'état civil et n'impose pas de transition physique. En cas de doute, l'officier d'état civil peut saisir le procureur de la République. Dans un deuxième temps, la mention du genre peut être modifiée devant le tribunal de grande instance. Elle concerne « toute personne majeure ou mineure émancipée qui démontre par une réunion suffisante de faits que la mention relative à son sexe dans les actes de l'état civil ne correspond pas à celui dans lequel elle se présente et dans lequel elle est connue ». Aucun prérequis médical ou chirurgical n'est dorénavant nécessaire. En France, l'assurance maladie prend en charge, sous certaines conditions, les dépenses de santé relatives à la dysphorie de genre.

\section{Transition physique}

L'évolution des personnes transgenres passe généralement par une transition physique qui peut comporter plusieurs étapes. En plus de la modification du style vestimentaire et de l'aspect physique, des interventions médicales et chirurgicales sont possibles.

Le traitement médical consiste en une hormonothérapie qui entraine la suppression des caractéristiques sexuelles du genre de départ, généralement associée à un traitement hormonal 
71 permettant l'évolution des caractéristiques physiques vers le genre dans lequel se reconnait 72 l'individu (Gender-affirming hormone treatment (GAHT)). La chirurgie plastique et la chirurgie de réassignation, sans être systématiques, sont également des options thérapeutiques. Les traitements hormonaux, bien que considérés comme réversibles, mais surtout les chirurgies entrainant la suppression des gonades, ont un impact sur la fertilité des personnes qui y ont recours. La dimension « reproductive » ou le désir de parentalité chez les personnes transgenres a longtemps été ignoré. Néanmoins, cet aspect est important à prendre en compte d'autant que les traitements médicaux ou les chirurgies peuvent être entrepris chez des adolescents ou de très jeunes adultes. Une préservation de la fertilité doit donc être discutée avant le démarrage de la transition physique médicale.

Dans cette revue, seront développées les indications et les modalités de préservation de la fertilité des femmes transgenres, les possibilités de restauration de la fertilité et la place de ces procédures dans le parcours de transition.

\section{Préservation de la fertilité des femmes transgenres (MtF)}

Indications

L'évolution de l'accompagnement médical des personnes transgenres permet actuellement de

91 proposer la mise en place de stratégies de préservation de la fertilité avant instauration des traitements hormonaux et/ou d'actes chirurgicaux. Un certain nombre de sociétés savantes, dont l'ASRM (American Society for Reproductive Medicine) ont émis des recommandations sur l'information concernant le possible impact des traitements sur les fonctions de reproduction et la possibilité d'avoir recours à la préservation de la fertilité [4]. 
En France, les recommandations de la W-PATH (World Professional Association of

97 Transgender Health) [5] ainsi que l'avis favorable rendu par le Défenseur des Droits en 2016 [2], ont conduit certaines équipes à proposer des parcours de préservation de la fertilité pour les personnes envisageant de s'engager dans un parcours de transition. Chez les personnes MtF,

100 les traitements hormonaux peuvent avoir un impact, encore mal défini mais potentiellement 101 délétère, sur la fertilité. La préservation de gamètes peut donc être proposée dans ce contexte, 102 en accord avec la loi bioéthique de 2011 [6].

\section{Les traitements hormonaux}

Pour les femmes transgenres, un traitement de «dévirilisation » qui permet l'atténuation des caractères sexuels secondaires est initié. Ce traitement consiste généralement en une hormonothérapie qui entraine la suppression des caractéristiques sexuelles du genre de départ. L'administration de progestatifs anti-androgènes freine la production de testostérone par effet anti-gonadotrope. L'acétate de cyprotérone (Androcur $\left.{ }^{\circledR}\right)$ était couramment prescrit en première

111 intention, mais étant donné le risque de méningiome associé à ce traitement, d'autres molécules

112 sont proposées telles que la spironolactone (100 à $200 \mathrm{mg} / \mathrm{j})$ ou des analogues de la GnRH 113 (Decapeptyl ${ }^{\circledR}(3,75 \mathrm{mg} / \mathrm{mois}(\mathrm{sc}))$. Ces molécules sont généralement associées à une 114 supplémentation en œstrogènes (17-bêta-oestradiol (E2), par prise orale (2 à $6 \mathrm{mg} / \mathrm{j}$ ) ou 115 transdermique $(0,1$ à $0,4 \mathrm{mg}$ deux fois par semaine) [7].

116 Les modifications physiques interviennent dans les 3 à 6 mois après l'instauration du traitement, 117 avec un effet maximal obtenu 4 à 5 ans plus tard. Les principaux effets observés sont une 118 redistribution du tissu adipeux, une augmentation du tissu mammaire, une diminution de la 119 pilosité faciale et corporelle et une diminution de la libido [8]. 
123 L'impact des traitements hormonaux sur la fonction testiculaire des femmes transgenres a été

124 peu étudié. Les études évaluant l'impact des traitements anti-androgènes ou des traitements 125 inhibant l'axe hypothalamo-hypophysaire peuvent apporter quelques réponses. Ainsi, les

126 études sur la contraception masculine ont mis en évidence que le blocage de la sécrétion de 127 gonadotrophines entrainait un arrêt de la spermatogénèse [9] qui pouvait être réversible en un 128 peu plus de 3 mois [10]. Concernant plus spécifiquement les traitements prescrits dans le cadre

129 d'une dysphorie de genre, très peu de données sont disponibles. Jusqu'en 2015, quelques séries

130 de cas évaluant l'histologie testiculaire après chirurgie ont rapporté une diminution majeure de

131 la spermatogénèse chez les femmes transgenres sous traitement hormonal. Cependant, dans

132 certaines situations, une spermatogénèse complète pouvait être observée [8]. En 2015, une

133 étude plus large incluant 108 patientes a été réalisée et a conclu à une histologie testiculaire très

134 hétérogène, quelle que soit la stratégie de traitement [11]. Néanmoins, une spermatogénèse a

135 essentiellement été retrouvée chez les femmes qui avaient arrêté le traitement hormonal 6

136 semaines avant l'intervention chirurgicale. Une supplémentation uniquement à base

137 d'œstrogènes pourrait aussi être un facteur limitant l'altération de la spermatogénèse.

139 Il reste encore beaucoup d'interrogations sur la réversibilité de ces traitements. Celle-ci dépend 140 probablement des durées, doses et types d'hormones déjà prescrites, ainsi que de facteurs

141 individuels, mais elle est vraisemblablement au minimum de 3 mois (un cycle complet de 142 spermatogénèse) [12]. Une amélioration des paramètres spermatiques chez les femmes 143 transgenres effectuant une fenêtre thérapeutique peut être espérée [13]. Cependant, une fenêtre 144 thérapeutique dans l'objectif d'obtenir le redémarrage de la spermatogénèse peut être difficile 145 à entreprendre pour les personnes supplémentées depuis longtemps. Les effets redoutés sont les 
146 modifications physiques et psychologiques qu'entrainerait l'arrêt du traitement. La réapparition

147 des caractéristiques masculines peut être vécue comme un retour en arrière. De plus, la 148 récupération de la spermatogénèse pourrait ne pas être systématique d'où l'importance de

149 proposer une préservation de la fertilité avant l'initiation des traitements.

Autoconservation de gamètes pour les personnes MtF (male to female) : aspect clinico-

152

153

154

155

156

157

158

159

160

biologique

La préservation de la fertilité chez les femmes transgenres repose essentiellement sur l'autoconservation de spermatozoïdes éjaculés. La congélation de ces cellules est une procédure simple et efficace. Un recueil de sperme est réalisé par masturbation, dans un laboratoire autorisé pour l'activité de préservation de fertilité, et les spermatozoïdes protégés par des cryoprotecteurs sont ensuite congelés dans des paillettes et conservés dans des containers d'azote à $-196^{\circ} \mathrm{C}$. Il est important d'anticiper cette étape car le recueil par masturbation peut s'avérer difficile pour les femmes transgenres. Dans certaines situations, un prélèvement de tissu testiculaire pourrait être proposé pour obtenir les spermatozoïdes.

La préservation de la fertilité n'a été généralisée que récemment dans le parcours de transition des personnes transgenres. Les données de la littérature sont donc relativement récentes et peu nombreuses. La plupart des articles disponibles ont été publiés à partir de 2018, dont une majorité d'études réalisées aux États-Unis. Beaucoup d'études rapportent la place de la préservation de la fertilité dans le parcours de transition au travers de questionnaires et évaluent le niveau d'information donné aux jeunes femmes au début du parcours de transition [14]. Quelques études ont rapporté des séries ou des cas cliniques de patientes qui ont bénéficié d'une 
170 préservation de la fertilité, mais elles sont peu nombreuses et les effectifs sont plutôt réduits

171 [13-22] (Tableau 2).

172 Les informations apportées par ce tableau montrent que la question de la fertilité des femmes

173 transgenres n'était initialement pas prioritaire dans le parcours de transition. Cependant

174 l'augmentation du nombre de publications ces trois dernières années témoigne d'un intérêt

175 récent pour le sujet, ainsi que de la systématisation de l'information donnée sur les possibilités

176 de PF dans le parcours de soin, comme cela est recommandé par certaines sociétés savantes [4].

177

178 Concernant la question de la parentalité, la majorité des hommes ou des femmes transgenres

179 interrogés déclarent vouloir avoir des enfants, mais peu d'entre eux ont recours aux techniques

180 de préservation de la fertilité [17, 20,23]. Plusieurs arguments sont généralement avancés pour

181 expliquer l'absence de préservation de la fertilité. Le décalage du démarrage des traitements

182 liés aux procédures de préservation de la fertilité est une explication. Le coût des actes de

183 préservation de la fertilité, qui dans beaucoup de pays sont à la charge des patients, est

184 également un frein. L'orientation sexuelle ainsi que la possibilité ou non du partenaire de porter

185 une grossesse interviennent également dans la décision. Enfin, l'information délivré par les

186 professionnels de santé peut également orienter les personnes. Il est également rapporté que

187 certains patients transgenres ne sont pas particulièrement attachés à la notion de parentalité

188 biologique, et sont ouverts aux stratégies alternatives comme l'adoption.

189 Lorsque la transition démarre à un très jeune âge, la congélation de gamètes matures n'est pas

190 possible. Il existerait des alternatives comme la congélation de tissu gonadique. Mais compte

191 tenu de l'incertitude lié à la réutilisation de ces tissus, ces procédures sont peu proposées [23].

192 Chez les jeunes patients la pression familiale est un des motifs de consultation de préservation

193 de la fertilité. Toutefois, le désir d'enfant n'est pas forcément la préoccupation principale du

194 jeune patient. 
198 Certaines études ont publié les données spermatiques des femmes transgenres qui ont bénéficié

199 d'une autoconservation de spermatozoïdes (Tableau 3) [12, 13, 24-27]. Les publications sont 200 peu nombreuses et les effectifs assez limités. Néanmoins, on observe globalement une altération 201 importante des paramètres spermatiques chez les femmes qui prennent un traitement hormonal, avec un risque élevé d'azoospermie [13]. La seule patiente qui présente des paramètres spermatiques normaux était supplémentée uniquement en œstrogènes [13]. Quelques patientes ont entrepris une fenêtre thérapeutique de 3 à 6 mois environ. Elles présentaient des paramètres spermatiques légèrement moins bons, que les femmes transgenres qui n'avaient jamais pris d'hormones [13]. Pour la plupart des patientes, les valeurs restaient supérieures aux valeurs normales [28], mais dans un cas, il a été décrit une absence de production de spermatozoïdes 4 mois après l'arrêt des traitements [12].

Quelques études ont rapporté une augmentation de l'altération des paramètres spermatiques

210 chez les femmes transgenres qui n'avaient pas encore démarré les traitements hormonaux par 211 rapport à une population contrôle [24, 25, 27]. Des hypothèses peuvent être avancées afin 212 d'expliquer cette possible altération. L'augmentation de la température scrotale due au port de 213 vêtements serrés ou aux techniques de dissimulation du sexe est une explication plausible [29]. 214 La diminution de la fréquence voire l'absence d'éjaculation pourrait aussi être une cause de 215 diminution de la production spermatique [30]. Enfin, le stress psychologique que vivent 216 certaines personnes pourrait participer à la diminution des paramètres spermatiques [25]. 
L’utilisation des spermatozoïdes congelés se fait après décongélation d'une paillette, à l'aide

221 de techniques d'assistance médicale à la procréation (AMP). Il s'agit des inséminations intrautérine (IIU) ou de la fécondation in vitro (FIV) sans ou avec micro-injection (ICSI) selon le contexte.

224 De rares cas de réutilisation ont été rapportés dans la littérature. En 2014, un enfant est né après 225 IIU réalisées avec les spermatozoïdes cryoconservés chez un couple composé d'une femme 226 transgenre et d'une femme cisgenre [27]. En 2017, c'est avec une technique de FIV avec 227 utilisation des spermatozoïdes cryoconservés qu'un couple composé d'une femme transgenre 228 et d'une femme cisgenre ont pu être parents [31]. Enfin, une grossesse évolutive a été obtenue 229 à l'aide d'une technique d'ICSI avec des spermatozoïdes cryoconservés [26].

230 Néanmoins, les possibilités d'utilisation ultérieure des gamètes cryoconservés sont encore 231 restreintes et dépendent des réglementations en vigueur dans les différents pays. Si le 232 changement d'état civil a été réalisé, les possibilités de réutilisation sont, à ce jour, inexistantes en France (Tableau 4). Aspects psycho-sexologiques

Il est important de proposer une consultation psycho-sexologique dans le parcours de prise en charge en préservation de la fertilité des personnes transgenres. Celle-ci permet d'évaluer le 239 possible impact psychique de la prise en charge, afin de prévenir de potentiels effets délétères. 240 Pour les personnes amenées à entamer une fenêtre thérapeutique, un accompagnement 241 psychologique durant cette période peut être nécessaire. L'autre élément important de cette consultation va concerner la possibilité d'exprimer son désir d'enfant, désir longtemps souvent

243 inhibé. En effet, jusqu'en 2016, les personnes transgenres devaient apporter la 244 preuve « irréversible et médicale d'une transformation physique » pour pouvoir effectuer un 
changement de sexe à l'Etat Civil. Cette disposition de la loi se traduisait par une stérilisation

246 systématique des personnes dans le cadre de leurs parcours de transition. En 2016, le 247 changement réglementaire a ouvert la voie à l'expression de ce désir, ainsi qu'à la possibilité 248 d'envisager un accès à des procédures d'AMP. Enfin, les procédures d'autoconservation vont 249 impliquer les organes génitaux, parties du corps qui peuvent être vécues de façon très négative 250 par les personnes transgenres. Pour les personnes MtF, le recueil par masturbation peut s'avérer 251 difficile ; il peut être nécessaire de travailler sur des stratégies le rendant possible. Il est 252 démontré que ce travail d'accompagnement permet un meilleur vécu [32].

\section{Conclusion}

La dimension « reproductive » et le désir de parentalité chez les personnes transgenres sont de plus en plus intégrés dès le début des parcours de transition, mais l'information et la mise en place de stratégie de préservation de la fertilité devrait être plus systématique. Un certain nombre de barrières empêchent ou retardent encore l'accès à ces techniques.

261 Si les possibilités d'utilisation ultérieure des gamètes sont encore restreintes et dépendent des réglementations en vigueur dans les différents pays, la cryoconservation de gamète ou de tissu gonadique est une étape importante de l'accompagnement global des personnes transgenres. Il

264 est important de déconnecter la préservation de la fertilité et l'utilisation des gamètes en 265 assistance médicale à la procréation. Le travail en réseau est primordial dans cette activité, 266 incluant les praticiens d'endocrinologie, de chirurgie, de gynécologie, de biologie de la reproduction, de psychiatrie et les psychologues. 
Les auteurs remercient Alix Béranger et Marie Prades pour leur aide dans la rédaction de cet

271 article.

\section{Références bibliographiques}

274

275

276

277

278

279

280

281

282

283

284

285

286

287

288

289

290

291

292

293

294

295

296

297

298

299

300

301

302

303

304

305

306

307

308

309

310

311

312

313

1. Flores AR, Brown TNTPark AS (2016) Public Support forTransgender Rights: A Twentythree Country Survey. The Williams Institute.

2. De Cuypere G, Van Hemelrijck M, Michel A, et al (2007) Prevalence and demography of transsexualism in Belgium. Eur Psychiatry 22: 137-41.

3. Olson J, Schrager SM, Belzer M, et al (2015) Baseline Physiologic and Psychosocial Characteristics of Transgender Youth Seeking Care for Gender Dysphoria. J Adolesc Health 57: 374-80.

4. Ethics Committee of the American Society for Reproductive M (2015) Access to fertility services by transgender persons: an Ethics Committee opinion. Fertil Steril 104: 11115.

5. W-PATH https://www.wpath.org/media/cms/Documents/SOC\%20v7/SOC\%20V7 French.pdf.

6. LOI ${ }^{\circ}$ 2011-814 du 7 juillet 2011 relative à la bioéthique. JORF $n^{\circ} 0157$ du 8 juillet 2011 , texte $n^{\circ} 1: 11826$.

7. Hembree WC, Cohen-Kettenis P, Delemarre-van de Waal HA, et al (2009) Endocrine treatment of transsexual persons: an Endocrine Society clinical practice guideline. J Clin Endocrinol Metab 94: 3132-54.

8. Schneider F, Kliesch S, Schlatt S, et al (2017) Andrology of male-to-female transsexuals: influence of cross-sex hormone therapy on testicular function. Andrology 5: 873-880.

9. Bremner WJ (2012) Contraception for men: a breakthrough new approach. Cell 150: 667-8.

10. Liu PY, Swerdloff RS, Christenson PD, et al (2006) Rate, extent, and modifiers of spermatogenic recovery after hormonal male contraception: an integrated analysis. Lancet 367: 1412-20.

11. Schneider F, Neuhaus N, Wistuba J, et al (2015) Testicular Functions and Clinical Characterization of Patients with Gender Dysphoria (GD) Undergoing Sex Reassignment Surgery (SRS). J Sex Med 12: 2190-200.

12. Barnard EP, Dhar CP, Rothenberg SS, et al (2019) Fertility Preservation Outcomes in Adolescent and Young Adult Feminizing Transgender Patients. Pediatrics 144.

13. Adeleye AJ, Reid G, Kao CN, et al (2019) Semen Parameters Among Transgender Women With a History of Hormonal Treatment. Urology 124: 136-141.

14. Baram S, Myers SA, Yee S, et al (2019) Fertility preservation for transgender adolescents and young adults: a systematic review. Hum Reprod Update 25: 694-716.

15. Segev-Becker A, Israeli G, Elkon-Tamir E, et al (2020) Children and Adolescents with Gender Dysphoria in Israel: Increasing Referral and Fertility Preservation Rates. Endocr Pract 26: 423-428.

16. Wakefield BW, Boguszewski KE, Cheney D, et al (2019) Patterns of Fertility Discussions and Referrals for Youth at an Interdisciplinary Gender Clinic. LGBT Health 6: 417-421. 
17. Chen D, Kyweluk MA, Sajwani A, et al (2019) Factors Affecting Fertility Decision-Making

18. Chiniara LN, Viner C, Palmert M, et al (2019) Perspectives on fertility preservation and parenthood among transgender youth and their parents. Arch Dis Child 104: 739-744.

19. Bartholomaeus CRiggs DW (2020) Transgender and non-binary Australians' experiences with healthcare professionals in relation to fertility preservation. Cult Health Sex 22: 129-145.

20. Riggs DWBartholomaeus C (2018) Fertility preservation decision making amongst Australian transgender and non-binary adults. Reprod Health 15: 181.

21. Chen D, Simons L, Johnson EK, et al (2017) Fertility Preservation for Transgender Adolescents. J Adolesc Health 61: 120-123.

22. Nahata L, Tishelman AC, Caltabellotta NM, et al (2017) Low Fertility Preservation Utilization Among Transgender Youth. J Adolesc Health 61: 40-44.

23. Segev-Becker A, Jacobson R, Stein R, et al (2020) Women with Nonclassic Congenital Adrenal Hyperplasia Have Gender, Sexuality, and Quality-of-Life Features Similar to Those of Nonaffected Women. Endocr Pract 26: 535-542.

24. Li K, Rodriguez D, Gabrielsen JS, et al (2018) Sperm cryopreservation of transgender individuals: trends and findings in the past decade. Andrology 6: 860-864.

25. Marsh C, McCracken M, Gray M, et al (2019) Low total motile sperm in transgender women seeking hormone therapy. J Assist Reprod Genet 36: 1639-1648.

26. Jones CA, Reiter LGreenblatt E (2016) Fertility preservation in transgender patients. International Journal of Transgenderism 0: 1-7.

27. Hamada A, Kingsberg S, Wierckx K, et al (2015) Semen characteristics of transwomen referred for sperm banking before sex transition: a case series. Andrologia 47: 832-8.

28. WHO (2010) WHO Laboratory Manual for the Examination and Processing of Human Semen World Health Organization, Geneva.

29. Thonneau P, Bujan L, Multigner L, et al (1998) Occupational heat exposure and male fertility: a review. Hum Reprod 13: 2122-5.

30. AlAwaqi AHammadeh ME (2017) Sexual abstinence and sperm quality. international Journal of Women's Health and Reproduction Sciences 5: 11-17.

31. Broughton DOmurtag K (2017) Care of the transgender or gender-nonconforming patient undergoing in vitro fertilization. International Journal of Transgenderism 18: 372-375.

32. Armuand G, Dhejne C, Olofsson JI, et al (2017) Transgender men's experiences of fertility preservation: a qualitative study. Hum Reprod 32: 383-390. 


\begin{tabular}{|c|c|}
\hline TERMES & DEFINITIONS \\
\hline Transidentité & Identité de genre différente du genre assigné à la naissance \\
\hline $\begin{array}{l}\text { Personne transgenre ou } \\
\text { personne trans }\end{array}$ & $\begin{array}{l}\text { Personne qui ne s'identifie pas à son sexe de naissance et qui vit ou souhaite vivre } \\
\text { dans un genre différent de celui qui lui a été assigné }\end{array}$ \\
\hline Cisgenre & Personne dont l'identité de genre correspond au sexe assigné à la naissance. \\
\hline Non binarité & Genre non exclusivement masculin ou féminin \\
\hline Dysphorie de genre & $\begin{array}{l}\text { Terme médical utilisé pour décrire la souffrance ou la détresse de la } \\
\text { personne transgenre face à un sentiment d'inadéquation entre son sexe assigné et } \\
\text { son identité de genre. }\end{array}$ \\
\hline Transsexualisme & $\begin{array}{l}\text { Terme historiquement utilisé, mais plus utilisé aujourd'hui car associé à la } \\
\text { pathologie mentale dont était auparavant créditer les personnes transgenres }\end{array}$ \\
\hline $\begin{array}{l}\text { Femme trans }=\text { MtF } \\
(\text { Male to Female })= \\
\text { personne } \\
\text { transmasculine }=\text { fille } \\
\text { trans }\end{array}$ & $\begin{array}{l}\text { Désigne une personne assignée homme à la naissance, mais dont l'identité de } \\
\text { genre est féminine. }\end{array}$ \\
\hline $\begin{array}{l}\text { Un homme trans }=\text { FtM } \\
\text { Female to Male) }= \\
\text { personne transféminine } \\
=\text { garçon trans }\end{array}$ & $\begin{array}{l}\text { Désigne une personne assignée femme à la naissance, mais dont l'identité de } \\
\text { genre est masculine. }\end{array}$ \\
\hline
\end{tabular}

Tableau 1: Termes et définitions liées à la transidentité 


\begin{tabular}{|c|c|c|c|c|c|c|c|}
\hline $\begin{array}{l}\text { Auteur, date, journal, } \\
\text { pays }\end{array}$ & Type d'étude & Effectif MtF & Age & $\begin{array}{l}\text { Information sur } \\
\text { l'impact des } \\
\text { traitements }\end{array}$ & $\begin{array}{l}\text { Consultation de } \\
\text { PF }\end{array}$ & Réalisation de la PF & Méthode de PF \\
\hline $\begin{array}{l}\text { Segev-Becker } \text { et al., } \\
2020 \text { Endocrine Practice, } \\
\text { Israel [15] }\end{array}$ & $\begin{array}{l}\text { Étude } \\
\text { rétrospective } \\
(2013-2018)\end{array}$ & $\begin{array}{l}47 \\
9 \text { prépubères } \\
38 \text { pubères }\end{array}$ & $\begin{array}{l}7,9 \\
15,9+/-1,7\end{array}$ & $\begin{array}{l}47 / 47(100 \%) \\
9 / 9 \\
38 / 38\end{array}$ & $\begin{array}{l}\mathrm{ND} \\
14 / 38\end{array}$ & $14 / 14$ & Spermatozoïdes (ej) \\
\hline $\begin{array}{l}\text { Wakefield et al., } 2019 \\
\text { LGBT Health, } \\
\text { USA [16] }\end{array}$ & $\begin{array}{l}\text { Étude } \\
\text { rétrospective } \\
(2010-2017)\end{array}$ & 28 & $\begin{array}{l}17,0+/-3.2 \\
{[7-25]}\end{array}$ & $19 / 28(67,9 \%)$ & $7 / 19$ & $\begin{array}{l}3 / 7 \text { tentatives } \\
2 \text { PF réalisés }\end{array}$ & Spermatozoïdes (ej) \\
\hline $\begin{array}{l}\text { Barnard et al., } 2019 \\
\text { Pediatrics, } \\
\text { USA [12] }\end{array}$ & $\begin{array}{l}\text { Etude } \\
\text { rétrospective } \\
(2015-2018)\end{array}$ & ND & 17 [15-24] & $\begin{array}{l}\text { PF proposée avant } \\
\text { initiation } \mathrm{TH}\end{array}$ & 11 & 10 (dont 2 pendant FT) & Spermatozoïdes (ej) \\
\hline $\begin{array}{l}\text { Chen et al., 2019, LGBT } \\
\text { health, USA [17] }\end{array}$ & $\begin{array}{l}\text { Questionnaires } \\
\text { (2016-2017) }\end{array}$ & 6 & $\begin{array}{l}18,4+/-2,3[15- \\
24]\end{array}$ & $6 / 6$ & $4 / 6$ & $4 / 6$ & Spermatozoïdes (ej) \\
\hline $\begin{array}{l}\text { Chiniara et al., } 2019 \\
\text { Archives of disease } \\
\text { Childhood, Canada [18] }\end{array}$ & $\begin{array}{l}\text { Questionnaires } \\
(2016-2017)\end{array}$ & 15 & {$[12-18]$} & $15 / 15$ & $2 / 15$ & $0 / 15$ & l \\
\hline $\begin{array}{l}\text { Bartholomaeus \& Riggs, } \\
\text { 2019, Culture, health and } \\
\text { sexuality, Australie [19] } \\
+ \\
\text { Riggs \& Bartholomaeus, } \\
\text { 2018, Reproductive } \\
\text { health Australie [20] }\end{array}$ & $\begin{array}{l}\text { Questionnaires } \\
(2018)\end{array}$ & $\begin{array}{l}72 \\
(295 \mathrm{MtF}+ \\
\text { FtM) } \\
\\
97(409 \mathrm{MtF}+ \\
\text { FtM) }\end{array}$ & $\begin{array}{l}25 \text { (médiane) } \\
28.5+/ 11,2[18- \\
72]\end{array}$ & $\begin{array}{l}26 \%(\mathrm{MtF}+\mathrm{FtM}) \\
70 / 409(17,1 \%) \\
(\mathrm{MtF}+\mathrm{FtM})\end{array}$ & $16(\mathrm{MtF}+\mathrm{FtM})$ & $\begin{array}{l}26 \mathrm{PF}(8,8 \% \mathrm{MtF}+\mathrm{FtM}) \\
\text { Dont } 10 \text { sans information préalable } \\
28 \mathrm{PF}(7 \%)\end{array}$ & $\begin{array}{l}\text { ND } \\
\text { gamètes }(\mathrm{MtF}+\mathrm{FtM})(27) \\
\text { embryons }(\mathrm{MtF}+\mathrm{FtM})(1)\end{array}$ \\
\hline $\begin{array}{l}\text { Adeleye et al., 2019, } \\
\text { urology, USA [13] }\end{array}$ & $\begin{array}{l}\text { Etude } \\
\text { rétrospective } \\
(2012-2018)\end{array}$ & 30 & $18-40$ & $30 / 30$ & ND & $\begin{array}{l}30 \text { tentatives } \\
\text { (dont } 28 \text { congélations, } 1 \text { vasectomie } \\
\text { et } 1 \text { échec de recueil) }\end{array}$ & Spermatozoïdes (ej) \\
\hline $\begin{array}{l}\text { Chen et al., } 2017 \text {, } \\
\text { Journal of adolescent } \\
\text { health, USA [21] }\end{array}$ & $\begin{array}{l}\text { Etude } \\
\text { retrospective } \\
(2013-2016)\end{array}$ & 6 & {$[15-21]$} & $6 / 6$ & $6 / 6$ & $\begin{array}{l}5 / 6 \text { tentatives } \\
4 \mathrm{PF}\end{array}$ & Spermatozoïdes (ej) \\
\hline $\begin{array}{l}\text { Nahata et al., 2017, } \\
\text { Journal of adolescent } \\
\text { health, USA [22] }\end{array}$ & $\begin{array}{l}\text { Etude } \\
\text { retrospective } \\
(2014-2016)\end{array}$ & 23 & $\begin{array}{l}15.2 \text { (médiane) } \\
{[9-18]}\end{array}$ & $23 / 23$ & ND & $\begin{array}{l}2 \text { tentatives } \\
1 \mathrm{PF}+1 \text { absence de spermatozoïdes } \\
\text { congelables ( } 13 \text { ans) }\end{array}$ & Spermatozoïdes (ej) \\
\hline
\end{tabular}

Tableau 2 : Description des études faisant état de l'information de l'impact des traitements et de la préservation de la fertilité chez les femmes transgenres

$\mathrm{PF}$ : préservation de la fertilité ; FT : fenêtre thérapeutique, TH : traitement hormonal ; ej : éjaculé ; FtM : homme trans, MtF : femme trans 


\begin{tabular}{|c|c|c|c|c|c|c|c|c|}
\hline $\begin{array}{l}\text { Auteur, date } \\
\text { Journal } \\
\text { Pays }\end{array}$ & Type d'étude & $\begin{array}{l}\text { Traitements } \\
\text { avant recueil }\end{array}$ & $\begin{array}{l}\text { Age } \\
\text { médian } \\
\text { (Années) }\end{array}$ & Effectif & $\begin{array}{l}\text { Volume } \\
\text { (ml) } \\
\text { (médiane) }\end{array}$ & $\begin{array}{l}\text { Concentration } \\
\text { (Millions/ml) } \\
\text { (médiane) }\end{array}$ & $\begin{array}{l}\text { Mobilité } \\
\text { progressive } \\
(\%) \text { (médiane) }\end{array}$ & Commentaires \\
\hline $\begin{array}{l}\text { Adeley et al.,2019, } \\
\text { Urology } \\
\text { USA }\end{array}$ & $\begin{array}{l}\text { Étude } \\
\text { rétrospective } \\
(2012-2018)\end{array}$ & $\begin{array}{l}\text { Aucun } \\
\text { FT (3-6 mois) }{ }^{(1)} \\
\text { TH en cours }^{(2)}\end{array}$ & $\begin{array}{l}22,3 \\
31,3 \\
28,9\end{array}$ & 18 & $\begin{array}{l}2,7 \\
2,1 \\
0,9\end{array}$ & $\begin{array}{l}63,6 \\
39 \\
2,4\end{array}$ & $\begin{array}{l}51,5 \\
34,3 \\
15,6\end{array}$ & $\begin{array}{l}\text { (2) TH : } \\
\text { - 3/7 : azoospermie, volume } \\
\text { diminué } \\
-1 / 7: 0,1 \mathrm{M} / \mathrm{ml} \\
\text { - 2/7: OAT modéré } \\
\text { - 1/7 (TH : E2 seul) : } \\
\text { paramètres spermatiques } \\
\text { normaux }\end{array}$ \\
\hline $\begin{array}{l}\text { Barnard et al., } 2019 \\
\text { Pediatrics } \\
\text { USA }\end{array}$ & $\begin{array}{l}\text { Étude } \\
\text { rétrospective } \\
(2015-2018)\end{array}$ & $\begin{array}{l}\text { Aucun } \\
\text { FT }(4 \text { et } 5 \mathrm{~m})^{(3)}\end{array}$ & $\begin{array}{l}20 \\
\\
18,5 \\
18 \\
19 \\
\end{array}$ & $\begin{array}{l}8 \\
2 \\
1 \\
1 \\
\end{array}$ & $\begin{array}{l}3 \\
\\
1,8 \\
2 \\
1,7 \\
\end{array}$ & $\begin{array}{l}21 \\
36,5 \\
73 \\
0 \\
\end{array}$ & $\begin{array}{l}54 \\
28 \\
56 \\
0 \\
\end{array}$ & $\begin{array}{l}\text { - } 2 \text { patientes sans PF dans le } \\
\text { groupe sans TH }\end{array}$ \\
\hline $\begin{array}{l}\text { Li et al., } 2018 \\
\text { Andrology } \\
\text { USA }\end{array}$ & $\begin{array}{l}\text { Étude } \\
\text { rétrospective / } \\
\text { comparative } \\
(2006-2016)\end{array}$ & $\begin{array}{l}\text { Aucun } \\
\text { TH }\end{array}$ & $\begin{array}{l}24,1 \\
\text { (moyenne) }\end{array}$ & 78 & 3,6 & $\begin{array}{l}14,2 \\
\text { NA }\end{array}$ & $\begin{array}{l}61,7 \\
\text { NA }\end{array}$ & $\begin{array}{l}\text { - N'ont été inclues que les } \\
\text { femmes qui ont eu une PF } \rightarrow \\
\text { biais } \\
\text { - Pas d'information sur FT } \\
\text { - Paramètres spermatiques } \\
\text { moins bon comparés à groupe } \\
\text { témoin (PF masc) }\end{array}$ \\
\hline $\begin{array}{l}\text { Marsh et al., 2018, } \\
\text { JARG } \\
\text { USA }\end{array}$ & $\begin{array}{l}\text { Étude cas témoin } \\
\text { (date ND) }\end{array}$ & Aucun & 25 & 22 & 2,4 & 31,89 & 44 & $\begin{array}{l}\text { - Paramètres spermatiques } \\
\text { moins bon comparé à des } \\
\text { témoins fertiles }\end{array}$ \\
\hline $\begin{array}{l}\text { Jones et al., } 2016 \\
\text { Int J } \\
\text { Transgenderism } \\
\text { Canada }\end{array}$ & $\begin{array}{l}\text { Étude } \\
\text { rétrospective } \\
(2010-2014)\end{array}$ & $\begin{array}{l}\text { NA (Début du } \\
\text { TH : } 0,33 \mathrm{~m} \text { en } \\
\text { moyenne }\end{array}$ & $\begin{array}{l}26,4 \\
\text { (moyenne) }\end{array}$ & 11 & NA & 30 & 46 & $\begin{array}{l}\text { - Pas de distinction TH ou non } \\
\rightarrow \text { biais }\end{array}$ \\
\hline $\begin{array}{l}\text { Hamada et al., } \\
\text { 2014, Andrologia } \\
\text { USA/Belgique/ } \\
\text { Canada }\end{array}$ & $\begin{array}{l}\text { Étude } \\
\text { rétrospective } \\
(2003-2011)\end{array}$ & Aucun & 28 & 29 & NA & 35 & 45 & \\
\hline
\end{tabular}

Tableau 3 : Paramètres spermatiques des femmes transgenres qui ont bénéficié une préservation de la fertilité

$\mathrm{PF}$ : préservation de la fertilité ; FT : fenêtre thérapeutique, TH : traitement hormonal ; OAT : oligo-asthéno-tératozoospermie

(1) FT Estrogènes +/- anti androgène (Estrogènes ou œstradiol +/- Spironolactone progestérone ou finasteride)

(2) $\mathrm{TH}$ : Durée médiane sous traitement 30 mois

(3) Leuprolide acetate (6 mois) et Spironolactone + œstradiol (26mois) 


\begin{tabular}{|c|c|c|c|c|c|}
\hline \multirow{2}{*}{$\begin{array}{l}\text { Situation } \\
\text { conjugale }\end{array}$} & \multicolumn{3}{|c|}{ Possibilités du projet parental } & \multirow{2}{*}{$\begin{array}{l}\text { Législation } \\
\text { en France }\end{array}$} & \multirow{2}{*}{$\begin{array}{l}\text { Cas } \\
\text { particuliers }\end{array}$} \\
\hline & Spermatozoïdes & Ovocytes & Utérus & & \\
\hline \multirow{3}{*}{$\mathrm{MtF}+\mathrm{M}$} & $\begin{array}{l}\text { Spermatozoïdes } \\
\text { conservés }\end{array}$ & $\begin{array}{l}\text { Don } \\
\text { d'ovocyte }\end{array}$ & GPA & $\begin{array}{l}\text { Non } \\
\text { autorisé }\end{array}$ & \\
\hline & $\begin{array}{l}\text { Spermatozoïdes } \\
\text { du conjoint }\end{array}$ & $\begin{array}{l}\text { Don } \\
\text { d'ovocyte }\end{array}$ & GPA & $\begin{array}{l}\text { Non } \\
\text { autorisé }\end{array}$ & \\
\hline & $\begin{array}{l}\text { Don de } \\
\text { spermatozoïdes }\end{array}$ & $\begin{array}{l}\text { Don } \\
\text { d'ovocyte }\end{array}$ & GPA & $\begin{array}{l}\text { Non } \\
\text { autorisé }\end{array}$ & \\
\hline \multirow[t]{2}{*}{$\mathrm{MtF}+\mathrm{F}$} & $\begin{array}{l}\text { Spermatozoïdes } \\
\text { conservés }\end{array}$ & $\begin{array}{l}\text { Ovocytes } \\
\text { de la } \\
\text { conjointe }\end{array}$ & Conjointe & $\begin{array}{l}\text { Non } \\
\text { autorisé }\end{array}$ & \multirow{2}{*}{$\begin{array}{l}\text { Autorisé si } \\
\text { absence de } \\
\text { changement } \\
\text { d'état civil }\end{array}$} \\
\hline & $\begin{array}{l}\text { Don de } \\
\text { spermatozoïdes }\end{array}$ & $\begin{array}{l}\text { Ovocytes } \\
\text { de la } \\
\text { conjointe }\end{array}$ & Conjointe & $\begin{array}{l}\text { Non } \\
\text { autorisé }\end{array}$ & \\
\hline \multirow[t]{2}{*}{$\begin{array}{c}\mathrm{MtF} \\
\text { célibataire }\end{array}$} & $\begin{array}{l}\text { Spermatozoïdes } \\
\text { conservés }\end{array}$ & $\begin{array}{l}\text { Don } \\
\text { d'ovocyte }\end{array}$ & GPA & $\begin{array}{l}\text { Non } \\
\text { autorisé }\end{array}$ & \\
\hline & $\begin{array}{l}\text { Don de } \\
\text { spermatozoïdes }\end{array}$ & $\begin{array}{l}\text { Don } \\
\text { d'ovocyte }\end{array}$ & GPA & $\begin{array}{l}\text { Non } \\
\text { autorisé }\end{array}$ & \\
\hline \multirow[t]{2}{*}{$\mathrm{MtF}+\mathrm{FtM}$} & $\begin{array}{l}\text { Spermatozoïdes } \\
\text { conservés }\end{array}$ & $\begin{array}{l}\text { Ovocytes } \\
\text { du } \\
\text { conjoint } \\
\text { ou Don } \\
\text { d'ovocyte }\end{array}$ & $\begin{array}{l}\text { Grossesse par } \\
\text { le conjoint si } \\
\text { utérus } \\
\text { conservé ou } \\
\text { GPA }\end{array}$ & $\begin{array}{l}\text { Non } \\
\text { autorisé }\end{array}$ & \\
\hline & $\begin{array}{l}\text { Don de } \\
\text { spermatozoïdes }\end{array}$ & $\begin{array}{l}\text { Ovocytes } \\
\text { du } \\
\text { conjoint } \\
\text { ou Don } \\
\text { d'ovocyte }\end{array}$ & $\begin{array}{l}\text { Grossesse par } \\
\text { le conjoint si } \\
\text { utérus } \\
\text { conservé ou } \\
\text { GPA }\end{array}$ & $\begin{array}{l}\text { Non } \\
\text { autorisé }\end{array}$ & \\
\hline
\end{tabular}




\begin{tabular}{|c|c|c|c|c|}
\hline \multirow[t]{3}{*}{$\mathrm{MtF}+\mathrm{MtF}$} & $\begin{array}{l}\text { Spermatozoïdes } \\
\text { conservés }\end{array}$ & $\begin{array}{l}\text { Don } \\
\text { d'ovocyte }\end{array}$ & GPA & $\begin{array}{l}\text { Non } \\
\text { autorisé }\end{array}$ \\
\hline & $\begin{array}{l}\text { Spermatozoïdes } \\
\text { de la conjointe }\end{array}$ & $\begin{array}{l}\text { Don } \\
\text { d'ovocyte }\end{array}$ & GPA & $\begin{array}{l}\text { Non } \\
\text { autorisé }\end{array}$ \\
\hline & $\begin{array}{l}\text { Don de } \\
\text { spermatozoïdes }\end{array}$ & $\begin{array}{l}\text { Don } \\
\text { d'ovocyte }\end{array}$ & GPA & $\begin{array}{l}\text { Non } \\
\text { autorisé }\end{array}$ \\
\hline
\end{tabular}

Tableau 4 : Possibilités d'assistance médicale à la procréation pour les femmes transgenres en fonction de leur situation conjugale 